\title{
Article \\ Steric Effects on Space Electroosmotic Thrusters in Soft Nanochannels
}

\author{
Jiaxuan Zheng, Beinan Jia and Yongjun Jian *
}

check for updates

Citation: Zheng, J.; Jia, B.; Jian, Y. Steric Effects on Space Electroosmotic Thrusters in Soft Nanochannels. Mathematics 2021, 9, 1916. https:/ / doi.org/10.3390/math9161916

Academic Editor: Rami

Ahmad El-Nabulsi

Received: 7 July 2021

Accepted: 10 August 2021

Published: 11 August 2021

Publisher's Note: MDPI stays neutral with regard to jurisdictional claims in published maps and institutional affiliations.

Copyright: (c) 2021 by the authors. Licensee MDPI, Basel, Switzerland. This article is an open access article distributed under the terms and conditions of the Creative Commons Attribution (CC BY) license (https:/ / creativecommons.org/licenses/by/ $4.0 /)$.
School of Mathematical Science, Inner Mongolia University, Hohhot 010021, China; 31536015@mail.imu.edu.cn (J.Z.); jbn_9792@163.com (B.J.)

* Correspondence: jianyj@imu.edu.cn

\begin{abstract}
The influence of steric effects on the performances of space electroosmotic thrusters (EOTs) was numerically delineated in soft nanochannels for which its walls are covered with polyelectrolyte materials. The size effect of the ionic species, namely the steric effect, is neglected in many previous research studies, but it has vital influences on electrostatic potential and electroosmotic velocity, which is further introduced into the present study in order to understand and improve the exploration of nano electroosmotic thrusters with soft channels. The thruster's thrust, specific impulse, total input power, thruster efficiency and thrust-to-power ratio are computed based on finite difference methods. It is found that the thruster's thrust and specific impulse increase with the steric parameter while the efficiency and thrust-to-power ratio possess opposite trends due to the enhancement of Joule heating dissipation. For real situations with the consideration of ion size, although the thruster's thrust could be promoted, the efficiency is only $30-70 \%$, and the peak values of thrust-to-power ratio fade away.
\end{abstract}

Keywords: steric effects; electroosmotic thrusters; soft nanochannels; electroosmotic flows; ion size effects; thruster performances

\section{Introduction}

For the last few decades, new aerospace missions, such as the trajectory corrections of small satellites and the precise control of spacecrafts performing combined measurements [1], have created some demands for space propulsion systems that are high precision, low energy dissipation, small thrust and so on in. The demand is gradually satisfied by the miniaturization of space components, which has promoted the development of mi$\mathrm{cro} /$ nano thrusters in space industry and astronautic engineering. Hence, the micro/nano spacecrafts have been widely focused and investigated by many experts and scholars [2-4]. Diez et al. [5] firstly proposed a new research area where the characteristics of micro/nanofluidic electrokinetics are introduced into space propulsion research studies so as to provide the basis for a novel class of micro/nano electroosmotic thrusters. Huang and Huang [6] further combined nano electrokinetic thrusters with two-liquid electroosmosis flows and analyzed the propulsion performances of the thrusters with slippery wall boundaries. The above studies are bound up with the electrical double layer (EDL) formed by the interaction of electrolyte solution with surface charges on the channel walls. When an external electric field is applied to two ends of micro/nano channels, mobile ions in the EDL are actuated by the electrostatic body force so that the bulk liquid is moved with them due to viscosity of the fluid, which is well known as electroosmotic flow (EOF). There is a large body of research studies about EOF in micro/nano channels [7-11].

The electroosmotic flow through micro/nano channels with soft material walls has been further considered and examined owing to its inherent features such as electrical phenomena, electrophoretic mobility and electrokinetic transport [12-16]. When the walls of a channel are grafted with a polyelectrolyte layer (PEL) regraded as the fixed charge layer (FCL), the channel is called as soft channels. Barbati and Kirby [17] theoretically and experimentally investigated electrokinetic transport characteristics of charged and 
uncharged soft interfaces in microfluidic systems. Balasubramanian et al. [18] numerically explored the dynamics of viscoelastic fluid in soft microchannels under the effects of rational forces and found that the drag effects of soft layer resulted in the steady state of fluid flow. Moreover, steaming potential and electoviscous effects in soft nanochannels were studied by Chen and Das [19] who obtained numerical velocity results of the flow for a large range of electrostatic potential. Their fluid motion, however, is different from our electroosmotic flow; that is, the former is driven by the pure pressure. It is possible that the common pressure actuation will be introduced and applied in space micro/nano thrusters considering the generation of an induced electric potential field in the future. The soft channel includes Donnan potential and an additional drag force within the FCL which, according to the above research works, affects the distributions for the electric potential and the flow velocity in channels so that thruster performances generated by the fluid motion are further changed.

We had considered the influence of soft nanochannel on the electroosmotic thrusters and found that the thruster could achieve efficiency up to $90 \%$ and deliver thrust at about $0-20 \mu \mathrm{N}$ when the soft material was dense $[20,21]$. When the PEL is dense, its dielectric permittivity is lower than the bulk aqueous medium, resulting in the rearrangement of ions in the channel, which is denoted as the ion partitioning effect. The effects of ion partitioning on the electrokinetics in soft nanochannels were analyzed in order to offset the existing work results with the consideration of equal permittivity inside and outside the ECL [22]. A large number of articles dealt with such effects in various research studies [23-25]. In this paper, however, we have not added the ion partitioning effect to present thruster systems since this consideration is repetitive. Moreover, based on features of the soft material, soft nanochannels have been widely used in energy conversion systems, transistors and diodes, nanofluidic and nanoionic valves and cell membranes [26-28]. It is noted that, in these research studies about electroosmotic flow, the ions in the solution are regarded as point charges, and their volumes are neglected. A theoretical study on the electrophoresis of soft particles was made with the consideration of ion partitioning effects and volume exclusion due to the finite ion size [29]. In fact, the size effect of the ion has significant influences on electrical potential of EDL and velocity distribution of EOF, which is further introduced in the present study with a focus on nano electroosmotic thrusters with soft channels.

The size effect of the ionic species, which is named as steric effect, is determined by the steric factor $v$ that represents the mean volume of each ion, and it is written as $v=2 a^{3} c_{0}$ where $a$ is the effective ion size, and $c_{0}$ is the bulk concentration [30]. As the steric factor is zero, the ions are thought of as point charges without the consideration of the steric effect. Many experts and scholars have pay attention to the significance of the steric effect on the EDL and EOF, as well as various modified models [31,32]. Yazdi et al. [33] investigated the influences of the steric effect on the fluid flow in a rectangular microchannel and acquired the high electroosmotic velocity with a high flow behavior index of the shearthickening fluid. Sin and Kim [34] theoretically reported the ion size effect on electrostatic and electroosmotic features in soft nanochannels and observed that the potential and velocity could increase while the ion number density decreased when considering the steric effect. Xing and Jian [35] studied that the pressure and electroosmotically driven flow in a soft nanochannel by taking the steric effect into account, along with the boundary slip condition and constant charge density on the walls. Moreover, it was found that the energy conversion efficiency for non-Newtonian Fluids in narrow confinements could be enhanced under the steric effect [36].

Therefore, in this study, we numerically delineate the influence of the steric effect on space electroosmotic thrusters (EOTs) in soft nanochannels. The electric potential is described to account for the size effect of ions in equilibrium via numerically solving the modified Poisson-Boltzmann equation with constant charge density on the walls. The electroosmotic velocity is presented according to the Navier-Stokes equation with the Naiver slip boundary condition. Based upon the distributions of the electric potential and flow velocity, the variations of thruster performances are obtained and analyzed by finite 
difference methods. Our main purpose is not only to understand the reality that there are size effects of ions in the electrolyte solution but also to understand and improve the exploration of thruster performances with soft nanochannels [20,21].

\section{Mathematical Modeling}

As shown in Figure 1a,b, we provide a model of single electroosmotic thruster emitter in soft nanochannels considering the effect of ion size. The length of the thruster is $L$, the width is $W$ and the height is $2 H$. We assume that the length is much larger than both height and width, i.e., $L>>2 H$ and $L>>W$, so that the flow in the thruster can be approximated to the instance that the fluid is between two flat plates. The nanochannel is covered by an ion-penetrable charged polyelectrolyte layer (PEL) of thickness $d(<H)$. For the sake of convenience, the two-dimensional coordinate system is established in the center of the channel, as presented in Figure 1b. The direction of flow is in the $x$-axis direction, which is created by an applied electroosmotic body force with an external electrical field $E_{x}$ along the flow direction.
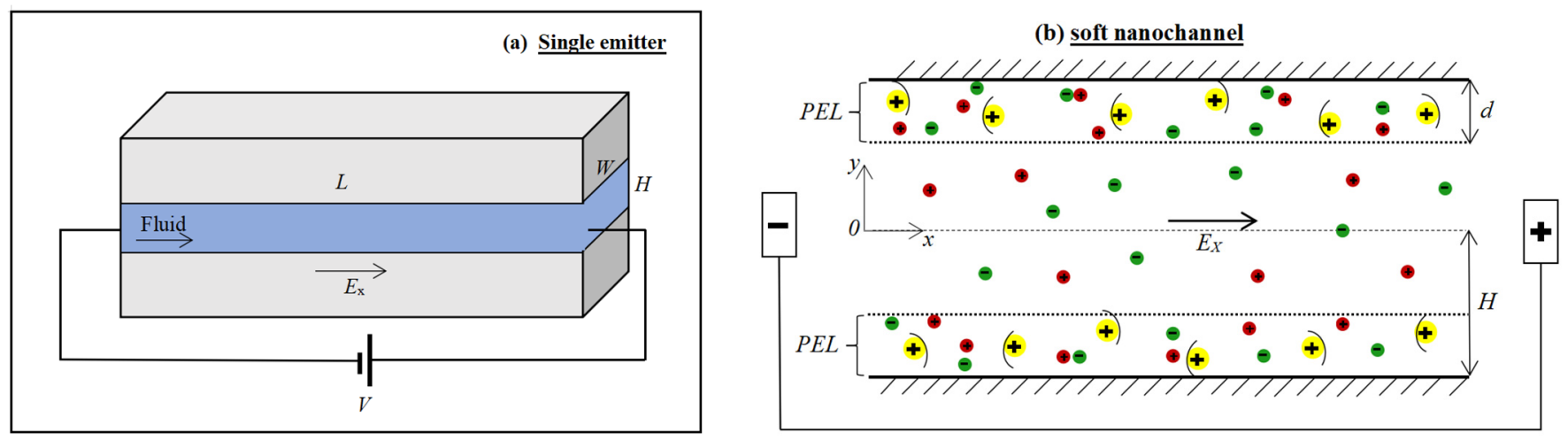

Figure 1. Schematics of (a) the single electrokinetic thruster emitter and (b) the soft nanochannel with three different kinds of ions, namely cations (red), anions (green) and FCL ions (yellow).

\subsection{Electric Potential Distribution}

As the potential distribution is symmetric about $y^{*}=0$, we consider only the top half of the channel $\left(0 \leq y^{*} \leq H\right)$. The Poisson-Boltzmann equation of electrical potential $\psi^{*}$ is expressed as follows [9].

$$
\begin{gathered}
\frac{d^{2} \psi^{*}}{d y^{* 2}}=-\frac{e z\left(n_{+}-n_{-}\right)}{\varepsilon},\left(0<y^{*}<H-d^{*}\right) \\
\frac{d^{2} \psi^{*}}{d y^{* 2}}=-\frac{e z\left(n_{+}-n_{-}\right)+Z e N}{\varepsilon},\left(H-d^{*}<y^{*}<H\right)
\end{gathered}
$$

The bulk fluid layer occupies the region $0<y^{*}<H-d^{*}$, and $H-d^{*}<y^{*}<H$ denotes the region where the FCL is present. Furthermore, $\varepsilon$ is the permittivity of the electrolyte, $e$ is electronic charge, and $Z$ and $N$ denotes the valence and the ionic number density of the FCL ions, respectively. Based on the assumption of uniform size and the symmetric valence of ions, $z$ represents valence number of ions, and $n_{+}$and $n_{-}$are the number densities of cations and anions in the electrolyte solution, respectively. Next, we consider the steric effect; thus, the Boltzmann distribution is written as follows [36]:

$$
n_{ \pm}=\frac{n_{0}}{1+2 v\left[\cosh \left(\frac{z e \psi^{*}}{k_{b} T}-1\right)\right]} \exp \left(\mp \frac{z e \psi^{*}}{k_{b} T}\right)
$$

where $n_{0}$ is the nominal ionic concentration at the reservoir, $k_{b}$ is the Boltzmann constant, $v$ is the steric factor, and $T$ is the average temperature. If the electrolyte solution carries with 
asymmetric ion sizes or asymmetric valence, the present Poisson-Boltzmann equation will be significantly revised [37-39]. By substituting (3) into (1) and (2), we rewrite them in the dimensionless form where these variables do not carry the asterisk *.

$$
\begin{gathered}
\frac{d^{2} \psi}{d y^{2}}=\frac{1}{\lambda^{2}} \frac{\sinh (\psi)}{1+2 v(\cosh (\psi)-1)},(0<y<1-d) \\
\frac{d^{2} \psi}{d y^{2}}=\frac{1}{\lambda^{2}} \frac{\sinh (\psi)}{1+2 v(\cosh (\psi)-1)}-\frac{1}{\lambda_{F C L}^{2}},(1-d<y<1)
\end{gathered}
$$

For more generality, the following dimensionless quantities are used:

$$
y^{*}=\frac{y}{H}, d^{*}=\frac{d}{H}, \psi^{*}=\frac{e z \psi}{k_{b} T}, \lambda^{*}=\frac{\lambda}{H}, \lambda_{F C L}^{*}=\frac{\lambda_{F C L}}{H}
$$

where $\lambda=\left[\left(\varepsilon k_{b} T\right) /\left(2 n_{0} e^{2} z^{2}\right)\right]^{1 / 2}$ is the EDL thickness, and $\lambda_{F C L}=\left[\left(\varepsilon k_{b} T\right) /\left(2 N e^{2} Z\right)\right]^{1 / 2}$ is the equivalent EDL thickness. Moreover, we have the following dimensionless boundary conditions:

$$
\left.\frac{d \psi}{d y}\right|_{y=0}=0,\left.\frac{d \psi}{d y}\right|_{y=(1-d)^{+}}=\left.\frac{d \psi}{d y}\right|_{y=(1-d)^{-}},\left.\psi\right|_{y=(1-d)^{+}}=\left.\psi\right|_{y=(1-d)^{-}},\left.\frac{d \psi}{d y}\right|_{y=1}=-\Omega
$$

where $\Omega=\omega e z H / \varepsilon k_{\mathrm{b}} T$ is the dimensionless surface charge density on the walls and, $\omega$ is surface charge density on the walls. We used the finite difference methods to solve Equations (4) and (5).

\subsection{Velocity Field Analysis}

We assume that the flow is a fully develop steady motion, and the pressure gradient is neglected in the electroosmotic thruster. This is an only pure electroosmotic flow problem. Therefore, the Navier-Stokes equations can be simplified as follows:

$$
\begin{gathered}
\mu \frac{d^{2} u^{*}}{d y^{*}}+e z\left(n_{+}-n_{-}\right) E_{x}=0,(0<y<1-d) \\
\mu \frac{d^{2} u^{*}}{d y^{*}}+e z\left(n_{+}-n_{-}\right) E_{x}-\mu_{c} u^{*}=0,(1-d<y<1)
\end{gathered}
$$

where $\mu$ is the dynamic viscosity of the solution, and $\mu_{\mathrm{c}}$ is the drag coefficient of the polyelectrolyte layer. The term $\mu_{\mathrm{c}} u$ is caused by the porosity that acts as a resisting force against the convective transport. In addition, the boundary conditions are provided as follows.

$$
\begin{gathered}
\left.\frac{d u^{*}}{d y^{*}}\right|_{y^{*}=0},\left.u^{*}\right|_{y^{*}=\left(H-d^{*}\right)^{+}}=\left.u^{*}\right|_{y^{*}=\left(H-d^{*}\right)^{-}} \\
\left.\frac{d u^{*}}{d y^{*}}\right|_{y^{*}=\left(H-d^{*}\right)^{+}}=\left.\frac{d u^{*}}{d y^{*}}\right|_{y^{*}=\left(H-d^{*}\right)^{-}},\left.u^{*}\right|_{y^{*}=H}=-\left.\gamma^{*} \frac{d u^{*}}{d y^{*}}\right|_{y^{*}=H}
\end{gathered}
$$

In the above equations, the following dimensionless quantities are introduced:

$$
u^{*}=\frac{u}{u_{H S}}, u_{H S}=\frac{\varepsilon k_{b} T E_{x}}{e z \mu}, \alpha=H \sqrt{\frac{\mu_{c}}{\mu}}, \gamma^{*}=\frac{\gamma}{H}
$$

where $u_{H S}$ is the Helmholtz-Smoluchowski electroosmotic velocity, $\gamma$ is the dimensionless slip coefficient, and $\alpha$ is the drag parameter. By substituting Equation (12) into Equations (8)-(11), the dimensionless Navier-Stokes equations and the boundary conditions can be written as follows.

$$
\frac{d^{2} u}{d y}-\frac{1}{\lambda^{2}} \frac{\sinh (\psi)}{1+2 v(\cosh (\psi)-1)}=0,(0<y<1-d)
$$




$$
\begin{gathered}
\frac{d^{2} u}{d y}-\frac{1}{\lambda^{2}} \frac{\sinh (\psi)}{1+2 v(\cosh (\psi)-1)}-\alpha^{2} u=0,(1-d<y<1) \\
\left.\frac{d u}{d y}\right|_{y=0},\left.u\right|_{y=(1-d)^{+}}=\left.u\right|_{y=(1-d)^{-}} \\
\left.\frac{d u}{d y}\right|_{y=(1-d)^{+}}=\left.\frac{d u}{d y}\right|_{y=(1-d)^{-}},\left.u\right|_{y=1}=-\left.\gamma \frac{d u}{d y}\right|_{y=1}
\end{gathered}
$$

The velocity distribution of fluid can be obtained by using numerical calculation.

\section{Thruster Performance Analysis}

\subsection{Thrust}

The propulsive forces of the thruster result from the generation of fluid motion from the outlet of the soft nanochannel. The thrust is deemed to be proportional to the mean flow velocity and mass flow rate of the fluid and can be analyzed via the variation in velocity. The expression of thrust is provided as follows $[6,20,40,41]$ :

$$
T h^{*}=2 W \int_{0}^{H} \rho u^{* 2}\left(y^{*}\right) d y^{*}=2 W \int_{H-d^{*}}^{H} \rho_{2} u_{2}^{* 2}\left(y^{*}\right) d y^{*}+\int_{0}^{H-d^{*}} \rho_{1} u_{1}^{* 2}\left(y^{*}\right) d y^{*}
$$

where $\rho_{1}$ and $\rho_{2}$ are assumed as the density of the bulk fluid layer and FCL layer, respectively. We can obtain the dimensionless form of the thrust as follows:

$$
T h=\int_{1-d}^{1} u_{2}^{2}(y) d y+\int_{0}^{1-d} \rho_{r} u_{1}^{2}(y) d y
$$

where $T h=T h^{*} /\left(2 W H \rho_{2} u_{H S}{ }^{2}\right)$ is the dimensionless thrust, and $\rho_{\mathrm{r}}=\rho_{1} / \rho_{2}$ is the density ratio of the two layers.

\subsection{Specific Impulse}

Specific impulse, $I_{s p}{ }^{*}$, describes the propellant efficiency of a thruster and is defined as the thrust divided by the mass flow of propellant. The specific impulse is normalized by the acceleration $g_{0}$ at the surface of Earth in order to improve comparability with different unit systems and is, therefore, measured in seconds. Thus, the specific impulse is represented as the ratio of propellant exhaust velocity to gravitational acceleration. Moreover, the propellant exhaust velocity is represented as the mean flow velocity. Hence, the specific impulse can be formulated as follows [6,20,41,42]:

$$
\begin{gathered}
I_{s p}^{*}=\frac{1}{g_{0} H} \int_{0}^{H} u^{*}\left(y^{*}\right) d y^{*}=\frac{1}{g_{0} H} \int_{H-d^{*}}^{H} u_{2}^{*}\left(y^{*}\right) d y^{*}+\int_{0}^{H-d^{*}} u_{1}^{*}\left(y^{*}\right) d y^{*} \\
I_{s p}=\int_{0}^{H} u(y) d y=\int_{1-d}^{1} u_{2}(y) d y+\int_{0}^{1-d} u_{1}(y) d y
\end{gathered}
$$

where $I_{s p}=I_{s p}{ }^{*}{ }^{*}{ }_{0} / u_{H S}$ is the dimensionless specific impulse.

\subsection{Efficiency}

Thruster efficiency is defined as the ratio of the power output to the power input $\left(P_{i n}{ }^{*}\right)$, and $P_{\text {in }}{ }^{*}$ includes the kinetic power $E_{k}{ }^{*}$, the Joule heating effect $P_{j}^{*}$, viscous dissipation $P_{v}{ }^{*}$ and frictional heating $P_{f}^{*}$. Moreover, the power output is supplied by the kinetic power 
$E_{k}{ }^{*}$. The kinetic power of the particle jet leaving the thruster is called jet power, and it is defined as follows $[6,20,40,41]$ :

$$
\begin{gathered}
E_{k}^{*}=\int_{A_{\text {out }}} \frac{1}{2} \rho u^{* 3} d A_{\text {out }}=W\left(\int_{H-d^{*}}^{H} \frac{1}{2} \rho_{2} u_{2}^{* 3} d y^{*}+\int_{0}^{H-d^{*}} \frac{1}{2} \rho_{1} u_{1}^{* 3} d y^{*}\right) \\
E_{k}=\frac{1}{2} \int_{1-d}^{1} u_{2}^{3} d y+\frac{1}{2} \int_{0}^{1-d} u_{1}^{3} d y
\end{gathered}
$$

where $E_{k}=E_{k}^{*} /\left(2 \rho H W u_{H S}{ }^{3}\right)$ is the dimensionless power output. The Joule heating effect, the viscous dissipation and the frictional heating are obtained in the following, respectively $[6,20,42]$ :

$$
\begin{gathered}
P_{j}^{*}=\int_{V} \sigma E_{x}^{2} d V=L W \int_{H-d^{*}}^{H}\left(\sigma_{0} \cosh \left(\frac{e z}{k_{b} T} \psi^{*}\right) E_{x}^{2} d y^{*}+\int_{H}^{H-d^{*}} \sigma_{0} \cosh \left(\frac{e z}{k_{b} T} \psi^{*}\right) E_{x}^{2} d y^{*}\right) \\
P_{j}=\beta\left(\int_{1-d}^{1} \cosh (\psi) d y+\int_{0}^{1-d} \cosh (\psi) d y\right) \\
P_{v}{ }^{*}=\int_{V} \mu\left(\frac{d u^{*}}{d y^{*}}\right)^{2} d V=L W\left(\int_{H-d^{*}}^{H} \mu\left(\frac{d u^{*}}{d y^{*}}\right)^{2} d y^{*}+\int_{0}^{H-d^{*}} \mu\left(\frac{d u^{*}}{d y^{*}}\right)^{2} d y^{*}\right) \\
P_{v}=\delta\left(\int_{1-d}^{1} \mu\left(\frac{d u}{d y}\right)^{2} d y+\int_{0}^{1-d} \mu\left(\frac{d u}{d y}\right)^{2} d y\right) \\
P_{f}^{*}=\left.\left.\int_{A_{\text {wall }}} \mu u^{*}\right|_{y^{*}=H} \frac{d u^{*}}{d y^{*}}\right|_{y^{*}=H} d A_{\text {wall }} \\
P_{f}=\left.\left.\delta u\right|_{y=1} \frac{d u}{d y}\right|_{y=1}
\end{gathered}
$$

where $\left[P_{j}, P_{v}, P_{f}\right]=\left[P_{j}^{*}, P_{v}^{*}, P_{f}^{*}\right] /\left(2 \rho H W u_{H S}{ }^{3}\right)$ is the dimensionless power, and $\beta=E_{x}^{2} L \sigma_{0} /\left(\rho u_{H S} 3\right)$ and $\delta=L \mu /\left(\rho H^{2} u_{H S}\right)$ are the characterized parameters of the Joule heating effect and frictional heating, respectively. Then, the efficiency is provided as $\eta=E_{k}{ }^{*} / P_{\text {in }}{ }^{*}$, where $P_{\text {in }}{ }^{*}$ is written as follows:

$$
\begin{gathered}
P_{i n}^{*}=E_{k}^{*}+P_{f}^{*}+P_{v}^{*}+P_{j}^{*} \\
P_{i n}=E_{k}+P_{f}+P_{v}+P_{j}
\end{gathered}
$$

where $P_{\text {in }}=P_{\text {in }}{ }^{*} /\left(2 \rho H W u_{H S}{ }^{3}\right)$ is the dimensionless power output.

\subsection{Thrust-to-Power Ratio}

Finally, thrust-to-power ratio is another significant parameter of space thrusters and can be acquired as follows: 


$$
\zeta^{*}=\frac{T h^{*}}{P_{i n}^{*}}, \zeta=\frac{T h}{P_{i n}}, \zeta=\zeta^{*} u_{H S}
$$

where $\zeta$ is dimensionless thrust-to-power ratio.

\section{Numerical Method}

In this paper, we use the finite difference method to obtain the electric potential distribution and velocity of fluid. The fundamental idea behind finite difference methods is to replace derivatives in the differential equation by discrete approximations and to perform evaluation on a grid to develop a system of equations. The boundary conditions are substituted in the system of equations where they are needed. We develop discrete approximations for the first derivative:

$$
y^{\prime}(x)=\frac{y(x+h)-y(x-h)}{2 h}
$$

and for the second derivative.

$$
y^{\prime \prime}(x)=\frac{y(x+h)-2 y(x)+y(x-h)}{h^{2}}
$$

In this paper, the Poisson-Boltzmann equation of electrical potential can be written as a system of equations after inserting the boundary conditions as follows.

$$
F(\psi)=\left[\begin{array}{c}
\frac{2 \psi_{2}-2 \psi_{1}}{h^{2}}-\frac{1}{\lambda^{2}} \frac{\sinh \left(\psi_{1}\right)}{1+2 v\left(\cosh \left(\psi_{1}\right)-1\right)} \\
\cdots \\
\frac{\psi_{i+1}-2 \psi_{i}+\psi_{i-1}}{h^{2}}-\frac{1}{\lambda^{2}} \frac{\sinh \left(\psi_{i}\right)}{1+2 v\left(\cosh \left(\psi_{i}\right)-1\right)} \\
\cdots \\
\frac{\psi_{N+1}-\psi_{N}}{h}-\frac{\psi_{N}-\psi_{N-1}}{h} \\
\cdots \\
\frac{\psi_{i+1}-2 \psi_{i}+\psi_{i-1}}{h^{2}}-\frac{1}{\lambda^{2}} \frac{\sinh \left(\psi_{i}\right)}{1+2 v\left(\cosh \left(\psi_{i}\right)-1\right)}+\frac{1}{\lambda_{F C L}^{2}} \\
\cdots \\
\frac{2 \psi_{n-1}-2 \psi_{n}-2 h \Omega}{h^{2}}-\frac{1}{\lambda^{2}} \frac{\sinh \left(\psi_{n}\right)}{1+2 v\left(\cosh \left(\psi_{n}\right)-1\right)}+\frac{1}{\lambda_{F C L}^{2}}
\end{array}\right]=0
$$

Moreover, we can obtain the Jacobin $D F(\psi)$ of $F(\psi)$. We utilize Newton's Method to solve the problem of the discretized version of the boundary value. The multivariate Newton's Method is the iteration $\psi^{\mathrm{k}+1}=\psi-D F\left(\psi^{k}\right)^{-1} F\left(\psi^{k}\right)$. The velocity of fluid can also be obtained by the same method.

After the substitutions, the system of equations can be solved by iterative methods. After the potential and velocity of the fluid are obtained, the thruster's thrust, specific impulse, total input power, thruster efficiency and thrust-to-power ratio are computed based upon composite trapezoid rule:

$$
\int_{a}^{b} f(x) d x=\frac{h}{2}\left(y_{0}+y_{m}+2 \sum_{i=1}^{m-1} y_{i}\right)
$$

where $y_{i}$ is the numerical solution of velocity $u_{i}$, which can be obtained by the finite difference method and Newton's Method. 


\section{Results and Discussion}

First of all, we used the finite difference methods to obtain the electric potential and the velocity distributions under the steric effect. Based on the fluid motion, these performances of thrusters, which are the thrust, specific impulse, efficiency as well as the thrust-to-power ratio and energy terms, are numerically explored for the different steric factors. The typical ranges of these relevant parameters in the study are provided as follows [43-46]: $H \sim 50 \mathrm{~nm} ; W \sim 100 \mathrm{~nm} ; L \sim 1 \mu \mathrm{m}$; viscosity of the liquid is $\mu \sim 10^{-3} \mathrm{~kg} /(\mathrm{ms})$; density of the liquid is $\rho \sim 10^{3} \mathrm{~kg} / \mathrm{m}^{3}$; relative permittivity is $\varepsilon_{r} \sim 78.36 \mathrm{~F} / \mathrm{m}$; permittivity of a vacuum is $\varepsilon_{0} \sim 8.854 \times 10^{-12} \mathrm{~F} / \mathrm{m}$; valence number of ions is $z \sim 1, \mathrm{~T} \sim 300 \mathrm{k} ; k_{b} \sim 1.381 \times 10^{-23} \mathrm{~J} / \mathrm{k}$; $e \sim 1.602 \times 10^{-19} \mathrm{C} ; n_{0}-\left(6.022 \times 10^{20}-10^{24}\right) / \mathrm{mol}$; the EDL thickness is $\lambda-(1-100) \mathrm{nm}$; steric factor is $v-(0-1)[35,47,48]$; electrical conductivity is $\sigma_{0}-\left(10^{-3}-10^{-1}\right) \mathrm{s} / \mathrm{m}$. Moreover, the applied axial electric field is set as $5 \times 10^{8} \mathrm{~V} / \mathrm{m}$ with a voltage drop between the electrodes of $500 \mathrm{~V}$ for a $1 \mu \mathrm{m}$ length channel, which is for producing the needed force and energy for space propulsion. The value of the voltage drop as high as 500-700 $\mathrm{V}$ has been used in molecular dynamic simulations of EOF for water solutions [5,6,20,21,40,41,48]. As the steric factor is zero, the problem becomes independent of the steric effect, which has been performed in previous studies [20]. Thus, we forced the problem to be dependent with the steric factor.

In order to illustrate the error between numerical and analytical solutions in soft nanochannels, we compared the non-dimension analytical solution of the velocity given by Zheng and Jian [20] with the present numerical solution when the steric effect is ignored $(v=0)$. Figure 2 shows that the numerical solution without consideration of the steric effect agrees well with the obtained analytical solution, which means that the current numerical method possesses correctness and feasibility.

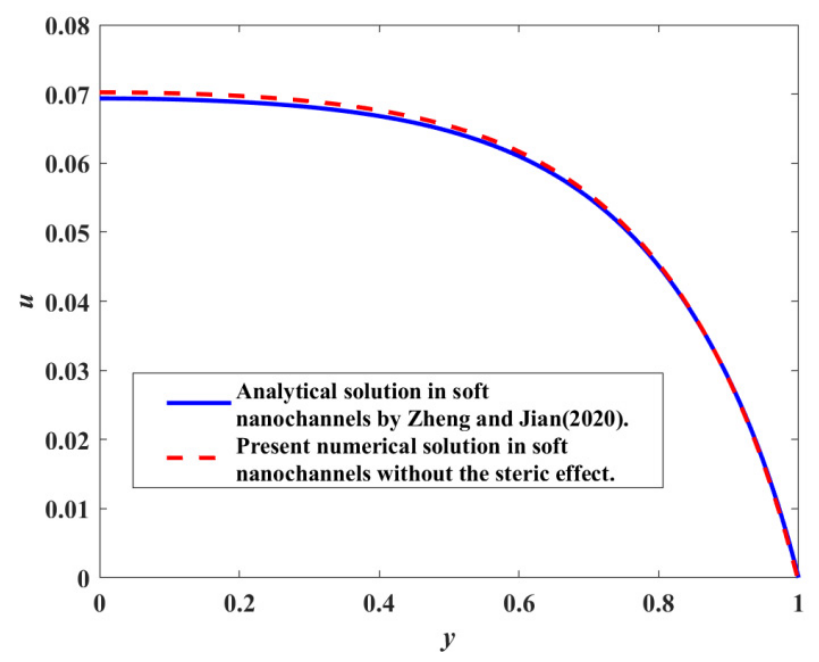

Figure 2. The comparison of the dimensionless analytical solution of velocity given by Zheng and Jian [20] with the present dimensionless numerical solution when the steric effect is ignored by setting the following parameters: $v=0, \lambda=0.2, \lambda_{F C L}=0.5, \alpha=1, \gamma=0$ and $\Omega=1$.

Figure 3 shows the variations of the dimensionless electrical potential and velocity with regard to different steric factors $v$. Compared with the case without the steric effect $(v=0)$, results show that the steric factor has significant influences on the electrical potential and velocity, and they increase with the steric factor. For the distribution of electric potential with the steric factor in Figure 3a, a physical explanation is that the net electrolyte charge density is equal to the fixed charge for the FCL at the point of electroneutrality, but the ion size effect results in the reduction in the net electrolyte charge content [49]. Hence, in order to ensure such the equilibrium, the corresponding potential has to increase. Moreover, the flow velocity increases with the steric factor in soft nanochannels owing to small values of $\lambda, \lambda_{F C L}$ and large surface charge density $\Omega$, which is chosen to generate enough flow 
speeds related to the needed thrust as well as energy for thrusters. When the channel walls carry with high charge density, the counter-ions adjacent to the wall will be firmly adsorbed due to strong electrostatic forces so that they cannot contribute to the movement of the fluid. With consideration of the ion size effect, however, the ions will be uniformly distributed across the channel due to the entropic effects so that the more "free" counterions are not absorbed and can assist movement of the fluid. Hence, the electroosmotic velocity augments under the steric effect. Moreover, if large values of $\lambda, \lambda_{F C L}$ and small $\Omega$ are considered, the variation of flow velocity will have a opposite trend. The results are same as the References $[35,47,50]$. Hence, it is necessary that influence of the steric effect on the performances of nano electroosmotic thrusters with soft channels is discussed in following figures.

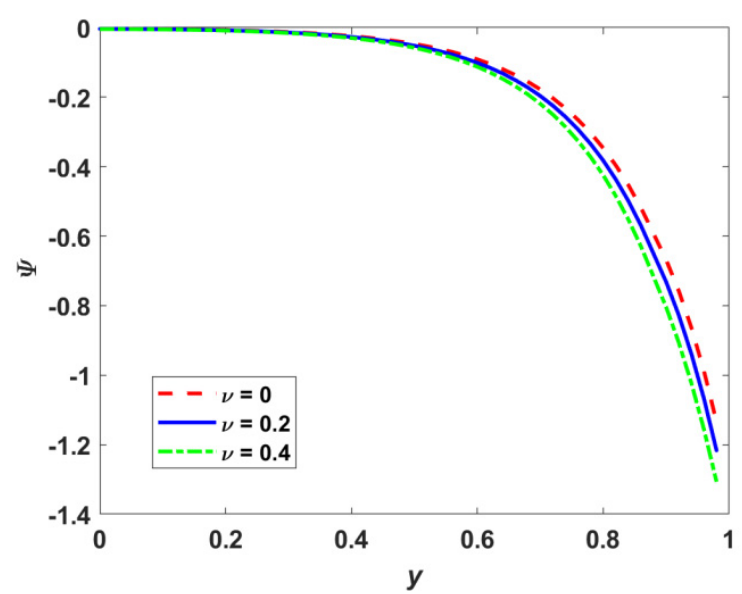

(a)

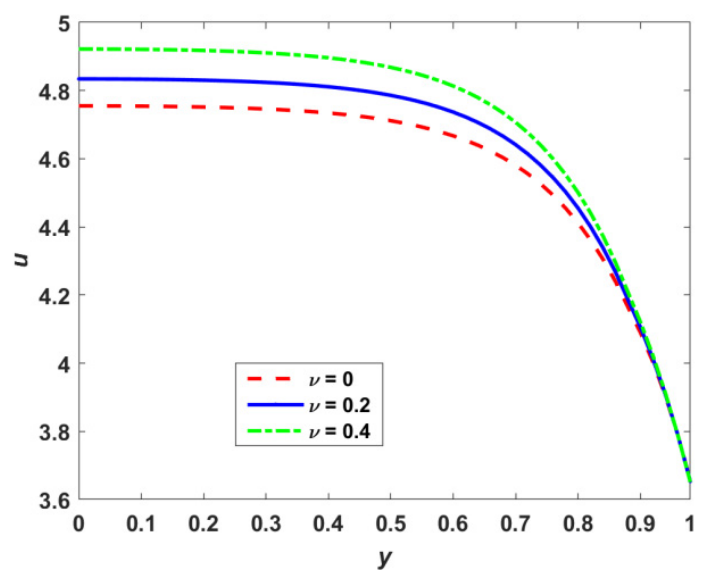

(b)

Figure 3. The distributions of (a) dimensionless electrical potential $\psi(y)$ and (b) dimensionless flow velocity $u(y)$ for the different steric factors $(v=0,0.2,0.4)$ when $\lambda=0.15, \lambda_{F C L}=0.5, \alpha=1, \gamma=0.5$ and $\Omega=8$.

Figure 4 presents the distributions of various thruster performances, namely (a) thrust, (b) specific impulse, (c) efficiency and (d) thrust-to-power ratio, with regard to different steric factors $v$, which are analyzed in dimension forms to intuitively show their variations and amounts. The variations of these performances with the slip length $\gamma$ are same with the results of Reference [20], when the steric effect is ignored $(v=0)$. If the ion size is considered in the study relative to the nano electroosmotic thrusters, it can well promote the enhancement of thrust; meanwhile, the specific impulse has a small amount of increase, as shown in Figure 4a,b. This is a good result since we increased the value of the steric factor in order to obtain more thrust while simultaneously not expending a lot of time. On the other hand, the efficiency of thrusters obviously reduces with the steric factor in Figure 4c. The cause is that the steric effect results in the increase in the electric potential so as to sharpen the enhancement of the Joule heating effect in Figure 5a, but the kinetic power and other dissipation items slowly increases with the steric factor, as shown in Figure $5 b, c$. According to the expression of the efficiency $\eta=E_{k}{ }^{*} / P_{\text {in }}{ }^{*}$, the denominator increases faster than the numerator does; thus, the efficiency reduces. In the last part of Figure 4, the influences of the steric factor on the thrust-to-power ratio are shown. The thrust-to-power ratio also decreases with the steric factor, which can be explained by Equation (31) that the power input, which is the denominator, increases faster than the thrust does, which is the numerator. That is to say that for the real situation with consideration of the ion size, the thruster thrust in soft nanochannels may be enhanced but the efficiency and thrust-to-power ratio obviously reduces owing to sharp enhancement of Joule heating dissipation. 


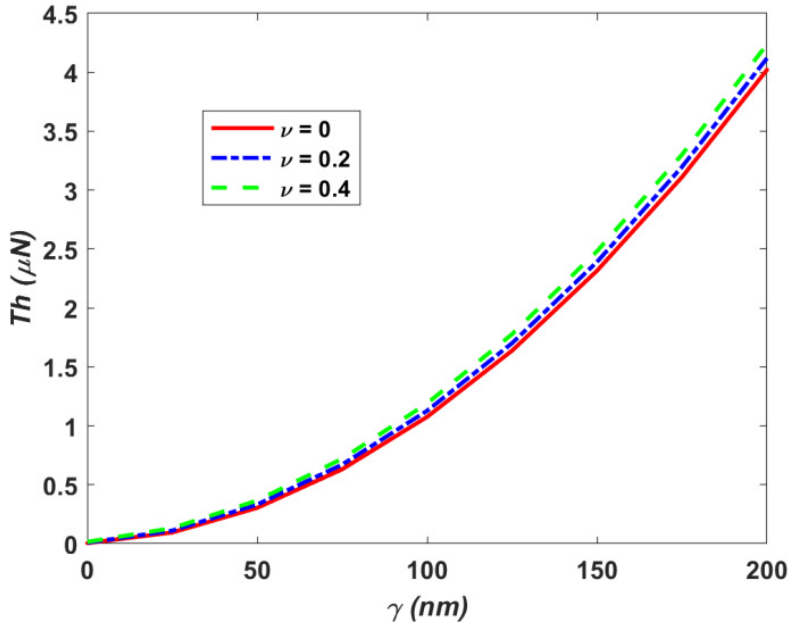

(a)

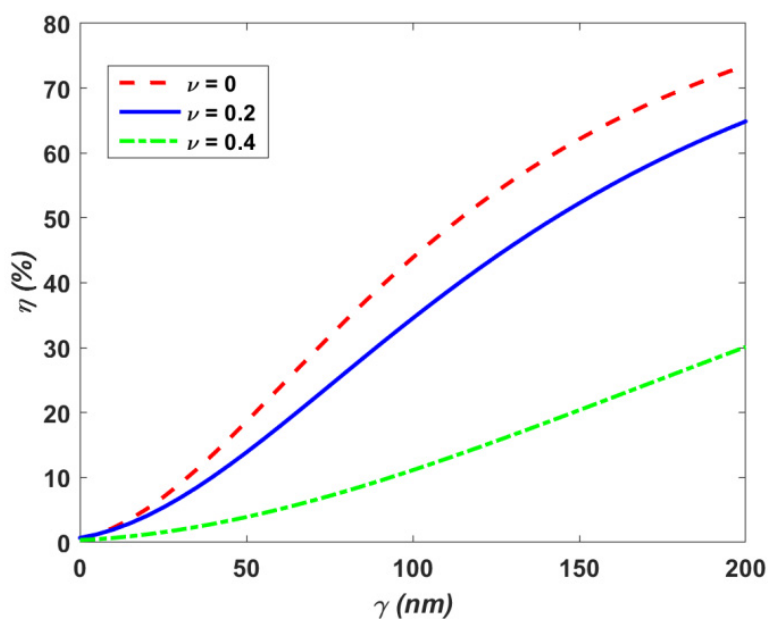

(c)

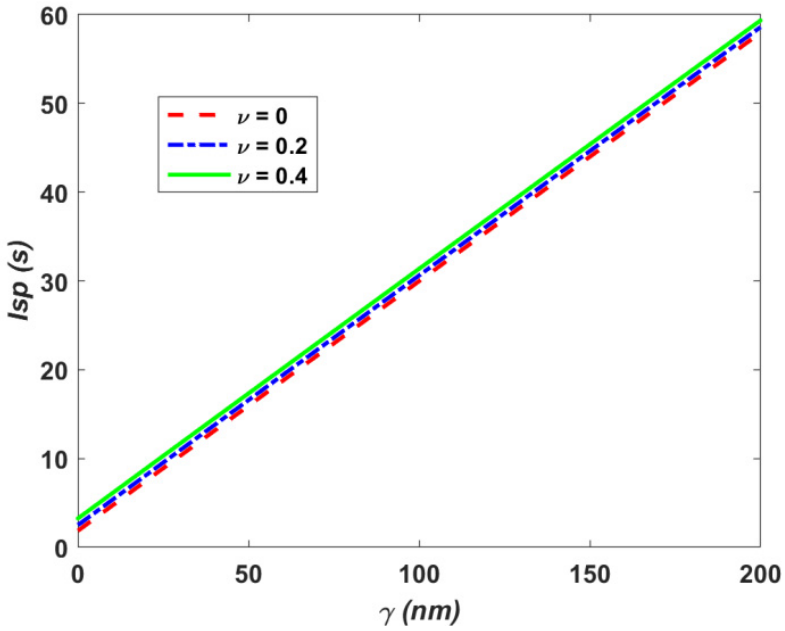

(b)

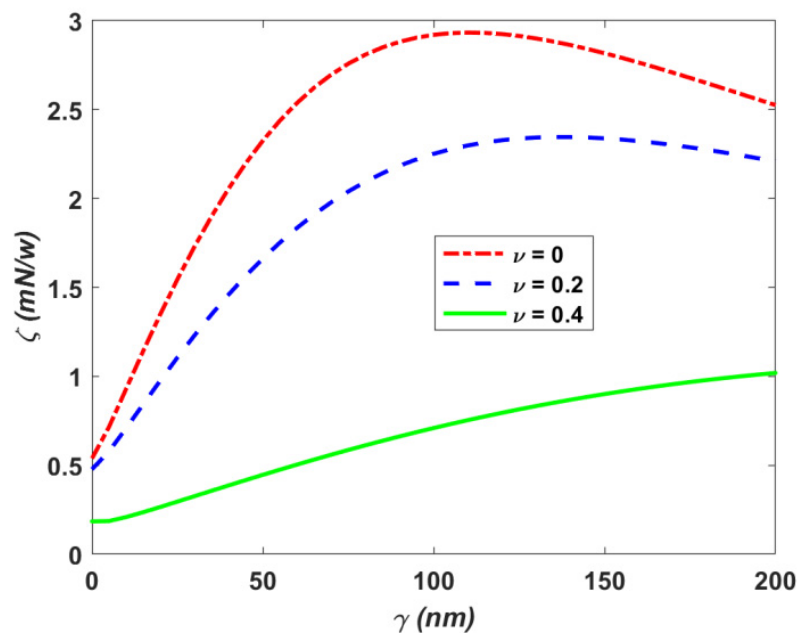

(d)

Figure 4. The variations of (a) thrust $T h^{*}$, (b) specific impulse $I_{s p^{*}}$, (c) efficiency $\eta$ and (d) thrust-to-power ratio $\zeta^{*}$ of the space electroosmotic thrusters with different steric factors $(v=0,0.2,0.4)$ when $\lambda^{*}=7.5 \mathrm{~nm}, \lambda^{*} F C L=25 \mathrm{~nm}, \mu_{c}=10^{4} \mathrm{~Pa} \mathrm{~s} /(\mathrm{km})^{2}$ and $\omega=5.4 \mathrm{mC} / \mathrm{m}^{2}$.

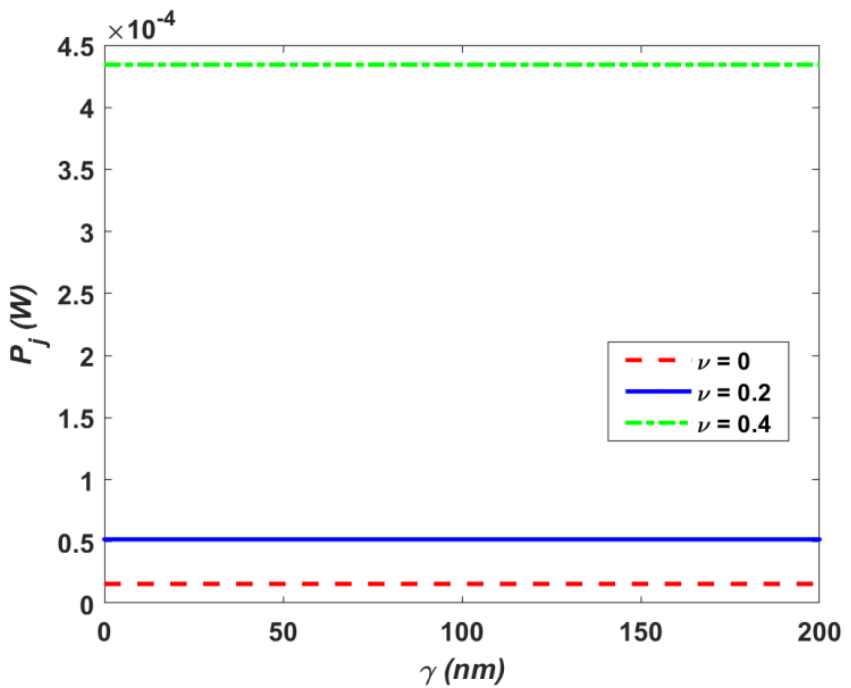

(a)

Figure 5. Cont. 


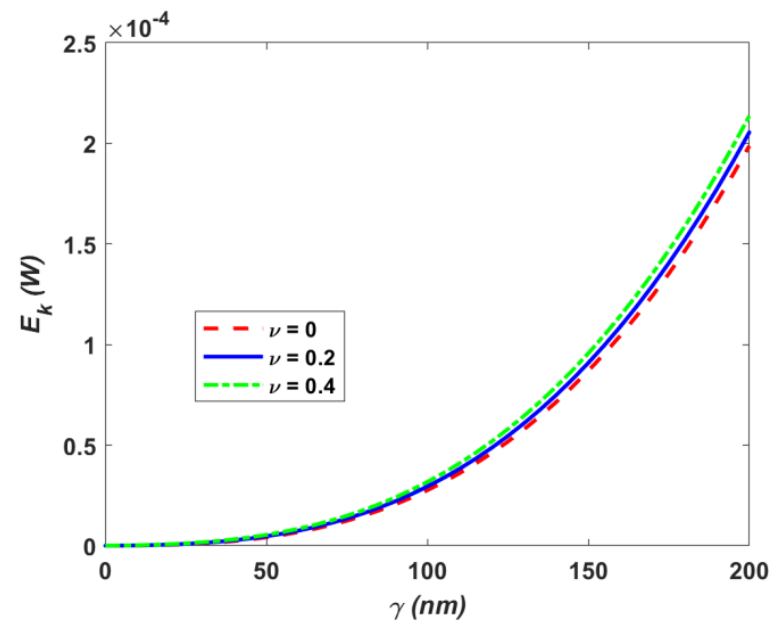

(b)

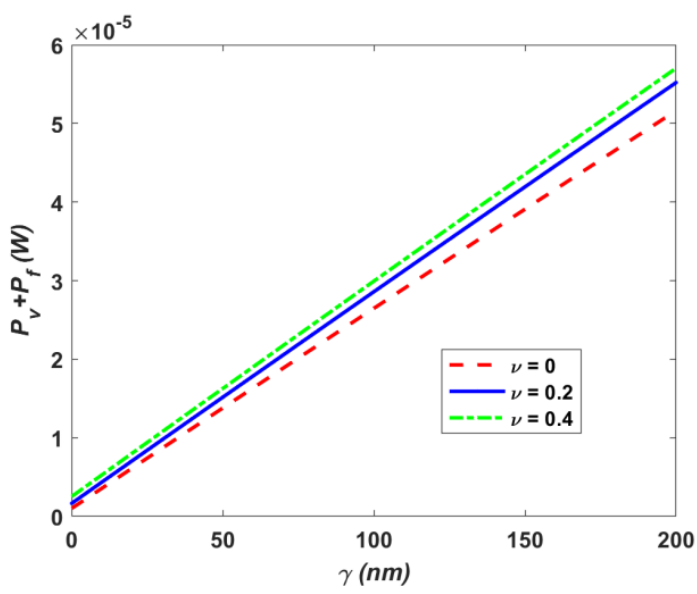

(c)

Figure 5. The variations of various energy items: (a) Joule heating effect $P_{j}^{*},(\mathbf{b})$ kinetic power $E_{k}{ }^{*}$ and (c) the sum of viscous dissipation $P_{v}{ }^{*}$ and frictional heating $P_{f}^{*}$ for different steric factors $(v=0,0.2,0.4)$ when $\lambda^{*}=7.5 \mathrm{~nm}$, $\lambda_{F C L}^{*}=25 \mathrm{~nm}, \mu_{\mathrm{c}}=10^{4} \mathrm{~Pa} \mathrm{~s} /(\mathrm{km})^{2}$ and $\omega=5.4 \mathrm{mC} / \mathrm{m}^{2}$.

\section{Experiment Design}

In this section, we designed three modeled electroosmotic thrusters (EOTs) shown in Table 1 with their detailed parameters and performances in order to draw a connection with the above-mentioned theoretical discussion on the influence of the steric effect on EOTs in soft nanochannels. The geometries of three of thrusters and the typical ranges of relevant parameters are same as those in Section 5. Moreover, we fix values of the surface charge density on the walls and the drag coefficient of the polyelectrolyte layer, namely $\omega=5.4 \mathrm{mC} / \mathrm{m}^{2}$ and $\mu_{\mathrm{c}}=10^{4} \mathrm{~Pa} \mathrm{~s} /(\mathrm{km})^{2}$. A comparison of thruster performances is presented with regard to the different steric factor as well as resultant parameters.

Table 1. The performance features of three single modeled electroosmotic thrusters in soft nanochannels, namely EOT 1 , $\mathrm{EOT}_{2}$ and $\mathrm{EOT}_{3}$, with consideration of the steric effect.

\begin{tabular}{|c|c|c|c|c|c|c|c|c|c|c|c|}
\hline \multirow[b]{2}{*}{ Design } & \multicolumn{4}{|c|}{ Parameters } & \multicolumn{7}{|c|}{ Single Thruster Performances } \\
\hline & $v$ & $\begin{array}{c}\lambda^{*} \\
(\mathrm{~nm})\end{array}$ & $\begin{array}{c}\lambda_{F C L}^{*} \\
(\mathbf{n m})\end{array}$ & $\gamma^{*}(\mathrm{~nm})$ & $I_{s p} *(\mathrm{~s})$ & $\begin{array}{c}T h^{*} \\
(\mu \mathrm{N})\end{array}$ & $\begin{array}{c}\eta \\
(\mathrm{mN} / \mathrm{W})\end{array}$ & $\zeta^{*}(\%)$ & $P_{j}^{*}(\mathrm{~mW})$ & $E_{k}^{*}(\mathrm{~mW})$ & $\begin{array}{l}P_{v}^{*}+P_{f}^{*} \\
(\mathrm{~mW})\end{array}$ \\
\hline $\mathrm{EOT}_{1}$ & 0 & 7.5 & 25 & 150 & 42.3 & 1.8 & 2.9 & 62 & $4.1 \times 10^{-3}$ & $3.9 \times 10^{-2}$ & $1.9 \times 10^{-2}$ \\
\hline $\mathrm{EOT}_{2}$ & 0.2 & 7.5 & 25 & 150 & 42.6 & 1.9 & 2.8 & 61 & $5.6 \times 10^{-3}$ & $4 \times 10^{-2}$ & $2 \times 10^{-2}$ \\
\hline $\mathrm{EOT}_{3}$ & 0.2 & 7.5 & 15 & 180 & 51.4 & 2.3 & 2.5 & 65 & $1.1 \times 10^{-2}$ & $7 \times 10^{-2}$ & $2.4 \times 10^{-2}$ \\
\hline
\end{tabular}

When ions in the electrolyte solution are deemed as point charges without consideration of steric effect, the performances of $\mathrm{EOT}_{1}$ are well obtained; that is, its thrust is about $0-10 \mu \mathrm{N}$, which satisfies the experiment requirement of a TianQin space detector [51], and the efficiency is observed to be up to $62 \%$, which is not bad. For the more real situation with consideration of the ion size $(v \neq 0)$, however, efficiency and thrust-to-power ratio of $\mathrm{EOT}_{2}$ significantly reduced. The reduction was able to be improved by adjusting other design parameters. For example, the efficiency of $\mathrm{EOT}_{3}$ is greater than those of $\mathrm{EOT}_{3}$ and $\mathrm{EOT}_{2}$ via enhancing slip length and decreasing equivalent EDL thickness, as shown in Table 1 . The cause is that the lower $\lambda^{*} F C L$ means the larger the ionic number concentration of ions in the FCL, and then the diving forces caused by the applied external electric field increases; thus, the thruster efficiency enhanced [20]. Moreover, it is a good result that the thrusts and specific impulses of $\mathrm{EOT}_{2}$ and $\mathrm{EOT}_{3}$ are increased under the steric effect compared with that of $\mathrm{EOT}_{1}$. 


\section{Conclusions}

In this research study, we investigated the influence of steric effects for the space electroosmotic thruster performances in soft nanochannels. After numerically obtaining distributions of the electric potential and flow velocity for the thruster, its thrust, specific impulse and total input power of (EOTs), the thruster efficiency and thrust-to-power ratio are calculated based on finite difference methods. The results show that the steric effect promotes enhancements of the thrust and specific impulse, but the efficiency and thrust-topower ratio significant declines. The reduction may be overcome by adjusting other design parameters, such as increasing the slip length or decreasing the equivalent EDL thickness.

In the future, we will change the electroosmotic-driven devices, for example, into the common pressure actuation for space micro/nano thrusters on the basis of the current research study, which is worth being explored.

Author Contributions: Conceptualization, Y.J., B.J. and J.Z.; methodology, Y.J.; software, B.J.; validation, B.J.; writing—original draft preparation, J.Z. and B.J.; writing—review and editing, Y.J., B.J. and J.Z.; supervision, Y.J. All authors have read and agreed to the published version of the manuscript.

Funding: This research study was funded by the National Natural Science Foundation of China (Grant No. 11772162) and the Inner Mongolia Grassland Talent (Grant No. 12000-12102408).

Institutional Review Board Statement: Not applicable.

Informed Consent Statement: Not applicable.

Data Availability Statement: Not applicable.

Acknowledgments: We would like to express our gratitude for the financial support from the National Natural Science Foundation of China (Grant No. 11772162), the Natural Science Foundation of Inner Mongolia Autonomous Region of China (Grant No. 2021MS01007) and the Inner Mongolia Grassland Talent (Grant No. 12000-12102408).

Conflicts of Interest: The authors declare no conflict of interest.

\section{References}

1. Smith, R.S. Control of deep-space formation-flying spacecraft, relative sensing and switched information. J. Guid. Control. Dyn. 2005, 28, 106-114. [CrossRef]

2. Mueller, J.; Chakraborty, I.; Bame, D.; Tang, W. Vaporizing liquid microthruster concept: Preliminary results of initial feasibility studies. Micropropuls. Small Spacecr. 2000, 187, 215-230.

3. Wright, W.P.; Ferrer, P. Electric micropropulsion systems. Prog. Aerosp. Sci. 2015, 74, 48-61. [CrossRef]

4. De Groot, W. Propulsion options for primary thrust and attitude control of microspacecraft. Microsatellites Res. Tools COSPAR Colloq. Ser. 1999, 10, 200-209.

5. Diez, F.J.; Hernaiz, G.; Miranda, J.J.; Sureda, M. On the capabilities of nano electrokinetic thrusters for space propulsion. Acta Astronaut. 2013, 83, 97-107. [CrossRef]

6. Huang, K.H.; Huang, H.F. Two-liquid electroosmotic thrusters for micro propulsion application. Phys. Fluids $2019,31,122003$.

7. Yang, R.J.; Fu, L.M.; Lin, Y.C. Electroosmotic flow in microchannels. J. Colloid Interface Sci. 2001, 239, 98-105. [CrossRef] [PubMed]

8. Xuan, X.C.; Li, D.Q. Electroosmotic flow in microchannels with arbitrary geometry and arbitrary distribution of wall charge. J. Colloid Interface Sci. 2005, 289, 291-303. [CrossRef]

9. Jian, Y.J.; Yang, L.G.; Liu, Q.S. Time periodic electroosmotic flow through a microannulus. Phys. Fluids 2010, 22, 04200. [CrossRef]

10. Bera, S.; Bhattacharyya, S. On mixed electroosmotic pressure driven flow and mass transport in microchannels. Int. J. Eng Sci. 2013, 62, 165-176. [CrossRef]

11. Ganguly, S.; Sarkar, S.; Hota, T.K.; Mishra, M. Thermally developing combined electroosmotic and pressure-driven flow of nanofluids in a microchannel under the effect of magnetic field. Chem. Eng. Sci. 2015, 126, 10-21. [CrossRef]

12. Ohshima, H. Dynamic electrophoretic mobility of a soft particle. J. Colloid Interface Sci. 2001, 233, 142-152. [CrossRef] [PubMed]

13. Ohshima, H. Electrophoretic mobility of a soft particle in a salt-free medium. J. Colloid Interface Sci. 2004, 269, 255-258. [CrossRef]

14. Ohshima, H. Theory of electrostatics and electrokinetics of soft particles. Sci. Technol. Adv. Mater. 2009, 10, 063001. [CrossRef]

15. Ohshima, H. Electrical phenomena in a suspension of soft particles. Soft Matter 2012, 8, 3511-3514. [CrossRef]

16. Kimiko, M.; Hiroyuki, O. Soft particle analysis of electrokinetics of biological cells and their model systems. Sci. Technol. Adv. Mater. 2011, 12, 023001.

17. Barbati, A.C.; Kirby, B.J. Soft diffuse interfaces in electrokinetics-theory and experiment for transport in charged diffuse layers. Soft Matter 2012, 8, 10598-10613. [CrossRef] 
18. Balasubramanian, S.; Kaushik, P.; Mondal, P.K. Dynamics of viscoelastic fluid in a rotating soft microchannel. Phys. Fluids 2020, 32, 112003. [CrossRef]

19. Chen, G.; Das, S. Streaming potential and electroviscous effects in soft nanochannels beyond Debye-Hückel linearization. J. Colloid Interface Sci. 2015, 445, 357-363. [CrossRef]

20. Zheng, J.X.; Jian, Y.J. Electroosmotic thrusters in soft nanochannels for space propulsion. Phys. Fluids 2020, 32, 122005. [CrossRef]

21. Zheng, J.X.; Jian, Y.J. Space Electroosmotic Thrusters in Ion Partitioning Soft Nanochannels. Micromachines 2021, 12, 777. [CrossRef]

22. Poddar, A.; Maity, D.; Bandopadhyay, A.; Chakraborty, S. Electrokinetics in polyelectrolyte grafted nanofluidic channels modulated by the ion partitioning effect. Soft Matter 2016, 12, 5968-5978. [CrossRef]

23. Mahapatra, P.; Gopmanda, P.P.; Duval, J.F.L. Effects of dielectric gradients-mediated ions partitioning on the electrophoresis of composite soft particles: An analytical theory. Electrophoresis 2021, 42, 153-162. [CrossRef]

24. Maurya, S.K.; Gopmandal, P.P.; Bhattacharyya, S.; Ohshima, H. Ion partitioning effect on the electrophoresis of a soft particle with hydrophobic core. Phys. Rev. E 2018, 98, 023103. [CrossRef]

25. Maurya, S.K.; Gopmandal, P.P.; Ohshima, H.; Duval, J.F.L. Electrophoresis of composite soft particles with differentiated core and shell permeabilities to ions and fluid flow. J. Colloid Interface Sci. 2020, 558, 280-290. [CrossRef] [PubMed]

26. Donath, E.; Voigt, A. Steaming current and streaming potential on structured surfaces. J. Colloid Interface Sci. 1986, 109, 122-139. [CrossRef]

27. Chanda, S.; Sinha, S.; Das, S. Steaming potential and electroviscous effects in soft nanochannels: Towards designing more efficient nanofluidic electrochemomechanical energy converters. Soft Matter 2014, 10, 7558-7568. [CrossRef] [PubMed]

28. Matin, M.H.; Ohshima, H. Combined electroosmotically and pressure driven flow in soft nanofluidics. J. Colloid Interface Sci. 2015, 460, 361-369. [CrossRef] [PubMed]

29. Gopmandal, P.P.; De, S.; Bhattacharyya, S.; Ohshima, H. Impact of ion-steric and ion-partitioning effects on electrophoresis of soft particles. Phys. Rev. E 2020, 102, 032601. [CrossRef] [PubMed]

30. Kilic, M.S.; Bazant, M.Z.; Ajdari, A. Steric effects in the dynamics of electrolytes at large applied voltages. I. Double-layer charging. Phys. Rev. E 2007, 75, 021502. [CrossRef]

31. Pandey, D.; Bhattacharyya, S. Influence of finite ion size and dielectric decrement on the ion current rectification in a single conical nanopore. Phys. Fluids 2021, 33, 062006. [CrossRef]

32. Kumar, B.; Gopmandal, P.P.; Sinha, R.K.; Ohshima, H. Electrophoresis of hydrophilic/hydrophobic rigid colloid with effects of relaxation and ion size. Electrophoresis 2019, 40, 1282-1292. [CrossRef] [PubMed]

33. Yazdi, A.A.; Sadeghi, A.; Saidi, M.H. Steric effects on electrokinetic flow of non-linear biofluids. Colloids Surf A Physicochem. Eng. Asp. 2015, 484, 394-401. [CrossRef]

34. Sin, J.-S.; Kim, U.-H. Ion size effect on electrostatic and electroosmotic properties in soft nanochannels with pH-dependent charge density. Phys. Chem. Chem. Phys. 2018, 20, 22961. [CrossRef]

35. Xing, J.N.; Jian, Y.J. Steric effects on electroosmotic flow in soft nanochannels. Meccanica 2018, 53, 135-144. [CrossRef]

36. Bandopadhyay, A.; Chakraborty, S. Steric-effect induced alterations in streaming potential and energy transfer efficiency of non-Newtonian fluids in narrow con-finements. Langmuir 2011, 27, 12243-12252. [CrossRef]

37. Sin, J.S.; Im, S.J.; Kim, K.I. Asymmetric electrostatic properties of an electric double layer: A generalized Poisson-Boltzmann approach taking into account non-uniform size effects and water polarization. Electrochim. Acta 2015, 153, 531-539. [CrossRef]

38. Kim, E.Y.; Kim, S.C. Electric double layer for a size-asymmetric electrolyte around a spherical colloid. J. Chem. Phys. 2014, 140, 154703. [CrossRef]

39. Gupta, A.; Rallabandi, B.; Stone, H.A. Diffusiophoretic and diffusioosmotic velocities for mixtures of valence-asymmetric electrolytes. Phys. Rev. Fluids 2019, 4, 043702. [CrossRef]

40. Goebel, D.M.; Katz, I. Fundamentals of Electric Propulsion: Ion. and Hall Thrusters; John Wiley \& Sons: Hoboken, NJ, USA, 2008.

41. Hey, F.G. Micro Newton Thruster Development; Springer: Berlin/Heidelberg, Germany, 2017.

42. Levine, S.; Marriott, J.R.; Neale, G.; Epstein, N. Theory of electrokinetic flow in fine cylindrical capillaries at high zeta-potentials. J. Colloid Interface Sci. 1975, 52, 136-149. [CrossRef]

43. Zhao, G.P.; Jian, Y.J. Thermal transport of combined electroosmotically and pressure driven nanofluid flow in soft nanochannels. J. Therm. Anal. Calorim. 2019, 135, 379-391. [CrossRef]

44. Xie, Z.Y.; Jian, Y.J. Rotating electroosmotic flow of power-law fluids at high zeta potentials. Colloids Surf. A Phys. Eng. Asp. 2014, 461, 231-239. [CrossRef]

45. Jian, Y.J.; Su, J.; Chang, L.; Liu, Q.S.; He, G.W. Transient electroosmotic flow of general Maxwell fluids through a slit microchannel. Z. Angew. Math. Phys. 2014, 65, 435-447. [CrossRef]

46. Das, S.; Chakraborty, S. Steric-effect-induced enhancement of electrical-double-layer overlapping phenomena. Phys. Rev. E 2011, 84, 012501.

47. Chanda, S.; Das, S. Effect of finite ion sizes in an electrostatic potential distribution for a charged soft surface in contact with an electrolyte solution. Phys. Rev. E 2014, 89, 012307. [CrossRef]

48. Joseph, S.; Aluru, N.R. Hierarchical multiscale simulation of electrokinetic transport in silica nanochannels at the point of zero charge. Langmuir 2006, 22, 9041-9051. [CrossRef] [PubMed]

49. Das, S. Electric-double-layer potential distribution in multiple-layer immiscible electrolytes: Effect of finite ion sizes. Phys. Rev. E 2012, 85, 012502. [CrossRef] 
50. Jimenez, E.; Escandón, J.; Méndez, F.; Bautista, O. Combined viscoelectric and steric effects on the electroosmotic flow in nano/microchannels with heterogeneous zeta potentials. Colloids Surf. A 2019, 577, 347-359. [CrossRef]

51. Luo, J.; Chen, L.-S.; Duan, H.-Z.; Gong, Y.-G.; Hu, S.; Ji, J.; Liu, Q.; Mei, J.; Milyukov, V.; Sazhin, M.; et al. TianQin: A space-borne gravitational wave detector. Class. Quantum Gravity 2016, 33, 035010. [CrossRef] 